\title{
A Survey on Sensors Lifetime Enhancement Techniques in Wireless Sensor Networks
}

\author{
Dhanashri V. Ambekar \\ Dept. of Electronics and \\ Telecommunication \\ G. H. Raisoni Institute of \\ Engineering and Technology \\ Pune, India
}

\author{
Amol D. Bhoi \\ Dept. of Electronics \\ G. H. Raisoni College of \\ Engineering \\ Nagpur, India
}

\author{
R. D. Kharadkar, Ph.D. \\ Dept. of Electronics \\ G. H. Raisoni Institute of \\ Engineering and Technology \\ Pune, India
}

\begin{abstract}
In the past years, Wireless Sensor Networks (WSNs) have gained increasing attention from both the users and researchers. It is used in various fields which include environmental, healthcare, military and other commercial applications. Sensor nodes are battery powered so energy constraints on nodes are very strict. When battery gets discharged, sensor node will get disconnected from remaining network. This results in link failure and data loss. In some applications battery replacement is also not possible. Hence energy efficient techniques should be designed which will enhance lifetime of network and accurate data transfer. In this paper, different sources of energy dissipation are listed followed by energy efficient techniques to enhance lifetime of the network.
\end{abstract}

\section{Keywords}

Wireless sensor networks, energy efficient techniques, lifetime of network

\section{INTRODUCTION}

The Advancement in wireless communication leads to develop wireless sensor networks (WSN). It consists of small devices. These devices collect information by coordinating with each other. These tiny devices are called as sensor node which consists of CPU (for data processing), memory (for data storage), battery (for energy) and transceiver (for receiving and sending signals or data from one node to another). The size of each sensor node varies with application requirement. For example, sensor nodes are microscopically small in military applications. The cost depends upon parameters like memory size, processing speed and battery [1]. Wireless sensor networks are used in commercial and industrial areas like habitat monitoring, environmental monitoring, healthcare, surveillance and process monitoring. We can also use it to monitor an activity such as physical and environmental conditions such as temperature, pressure, sound and pass their data to the sink which is the base station(called sink).A sensor node includes three basic components which are as follows:

a) A sensing subsystem for data acquisition from the physical surrounding environment.

b) A processing subsystem for local data processing and storage.

c) A wireless communication subsystem for data transmission.

The battery source supplies energy needed by the device to perform tasks. However, it consists of limited energy resource. It becomes very difficult to recharge the battery, because nodes may be placed in hostile or unpractical environment. Among the set of potential scenarios, applications with monitoring task benefit from this technology as WSNs allow a data acquisition at scales and resolutions that are difficult to achieve with previous techniques. Lifetime of a sensor network should be long enough to fulfill application requirement. External power supply shows a noncontinuous behavior so that energy buffer is needed. Therefore, energy conservation plays vital role in design of systems based on wireless sensor networks.

A WSN consists of a number of sensor nodes spread across a small or large geographical area. Each sensor node has wireless communication capability and significant intelligence for signal processing and networking of the data. AWSN can be placed in remote geographical locations [2]. It requires minimum amount of setup and administration costs. In WSNs the nodes act both as hosts and as routers. They operate in a self-organizing and adapting manner. Typical WSNs communicate directly with a centralized controller or a satellite, thus communication between the sensor and controllers is based on a single hop. In future, a WSN could be a collection of autonomous nodes that communicate with each other by forming a multi-hop network.

The use of WSN is increasing day by day and at the same time facing problem of energy constraints in terms of short battery lifetime. Every node depends on the battery source for various activities; this has becoming a major issue in wireless sensor networks. If anyone node among all the nodes gets failed, the entire system gets interrupted. Every sensor node can be in active, sleep and idle modes. In active state, receiving and transmission of data is carried out. In idle mode, the nodes consume same amount of energy as in active mode. While in sleep mode, nodes make radio to shutdown to save the energy. The following steps can be taken into consideration to save energy caused by communication in WSNs [3].

a) Schedule the various states of nodes (i.e. transmitting, receiving, idle or sleep).

b) Change in transmission range between the sensing nodes.

c) Use of efficient routing and data collecting methods.

d) Avoid the handling of unwanted data as in overhearing process.

In many situations, (e.g. Surveillance applications), it is not possible to replace the battery source which is drained of energy. Many researchers are trying to find power-aware protocols for wireless sensor networks in order to overcome 
energy efficiency problems. Protocols should provide real time support as they are applied in areas where data is sensed, processed and transmitted. If a protocol has a fast and reliable in reactions to changes in network, it is said to have real-time support. The delay in transmission of data from sensor nodes to sink should be less, which turns into fast response.

\section{CHALLENGES IN THE NETWORK DESIGN AND ROUTING ISSUES}

Several factors should be taken into consideration while designing a network and routing protocols. Some limitations of network resources affect WSNs, for example, energy, central processing unit, bandwidth and storage [4][5]. The challenges in design of sensor networks involve following main aspects[4][5][6]:

\subsection{Sensor Deployment}

While designing of routing protocols, one has to manage the locations of the sensors. Most of the protocols assume that the sensors are in built of GPS receivers or use some localization techniques to know about sensor positions.

\subsection{Limited Energy Capacity}

Since batteries are used as a power source in sensor nodes, they have limited energy capacity. In battlefield, as it is not possible to recharge batteries and access the sensors, energy constraints on sensors are tight. When energy of the sensor reaches defined threshold, it will not able to function properly. Hence, prolonged network lifetime can be achieved by designing energy efficient routing protocols for sensors.

\subsection{Data Aggregation}

Optimized data transfer as well as energy efficiency can be achieved by using data aggregation technique. The retransmissions of similar packets will be significantly reduced which helps in minimizing the redundant data.

\subsection{Various Sensing Application Requirements}

There are numerous applications in which sensor networks are used. A single network protocol cannot satisfy the requirements of all applications. Therefore routing protocols should be able to in accurate data delivery and sink can gather all information about physical phenomenon on time.

\subsection{Scalability}

In WSN, networks may be of different sizes and such networks may contain sensors with different capacities and asymmetric links between them. In such cases routing protocols should scale with these aspects of network.

\section{Energy conservation can be achieved at}

a) Device level: By appropriate selection and configuration of hardware.

b) Network level: By selecting communication methods and protocols.

The sensor node consists of processing unit, sensing unit, transceiver unit and power unit [7]. Processing unit can read data of sensor and also perform some calculations and make a packet ready to transfer in the channel. Sensor converts energy from one form to another form. Basically it acts as a transducer which converts energy into analog or digital signal. Sensors can be eminent based on kind of energy they detect or transfer to the system. Typical sensor node can be built with different types of sensors. Some of them need a large amount of energy than others.

\section{REASONS OF ENERGY WASTAGE}

In WSNs, energy is dissipated by sensors while sensing, processing, transmitting or receiving data to fulfill the mission required by the application. Data acquisition is done by sensing subsystem. The energy can be saved if data from transducer can be minimized. Communication system is a greedy source of energy dissipation. Great amount of energy is wasted in states that are useless from the application point of view such as [8]:

\subsection{Collision}

When a node receives more than one packet at the same time, collision will takes place between these packets. All packets that cause the collision should be discarded. And retransmission of these packets is required.

\subsection{Overhearing}

When a sender transmits a packet, all neighboring nodes in its transmission range receive the packet even if it is not destined for them. Thus the energy is wasted when a node receives packets which are not intended to be received.

\subsection{Idle Listening}

It is one of the major sources of energy wastage. Energy dissipation takes place when a node is listening to an idle channel in order to receive possible traffic.

\subsection{Interference}

The nodes located between transmission range and interference range receives a packet but cannot decode it.

Above mentioned reasons give us general idea about energy dissipation sources. As network lifetime becomes important factor for evaluating WSN, energy conservation techniques should be designed to enhance the network lifetime.

\section{CLASSIFICATION OF ENERGY EFFICIENT TECHNIQUES}

Energy efficient techniques can be classified in five main classes namely, data reduction, protocol overhead reduction, energy efficient routing, duty cycling and topology control.

\subsection{Data Reduction}

It mainly focuses on reducing the amount of data produced, processed and transmitted. Data compression and data aggregation uses data reduction technique.

\subsection{Protocol Overhead Reduction}

This technique is used to increase protocol efficiency by reducing the overhead. Optimized flooding is also used to reduce the overhead. Transmission periods of messages are selected depending upon the stability of the network, or on the distance to the source of the transmitted information.

\subsection{Energy Efficient Routing}

Routing protocols should be designed in such a way that network lifetime should be increased and energy consumed by end to end transmission should be minimized. Nodes with low residual energy should be avoided. Some protocols use geographical coordinates of nodes to build a route toward the destination. Others build a hierarchy of nodes to simplify routing and reduce overhead. Data centric protocols send data to only nodes which are interested to avoid useless transmissions. 


\subsection{Topology Control}

It adjusts transmission power and maintains network connectivity. It will reduce energy consumption.

\section{GENERAL APPROACHES FOR ENERGY SAVING}

Different methods can be used to reduce energy consumption in wireless sensor networks (WSN) such as,

\subsection{System Partitioning}

Sensor nodes in WSN have limited amount of energy as they are battery powered. The sensor nodes may be deployed in harsh environmental conditions depending upon the application requirement. Node failure is quiet common in defense applications where problem of malicious tempering; environmental degradation can affect performance of nodes. Due to which network topology may become disconnected. Due to failure of set of nodes, a subset of nodes that have not failed also becomes separated from rest of the network. This is called as "cut" in a network which results in loss of packet data. The technique is used which divides the sensor nodes into $\mathrm{N}$ sets to achieve the maximal lifetime improvement. System partitioning can be used to reduce energy consumption in the network.

\subsection{Dynamic Voltage Scaling}

To develop an energy efficient system is one of the challenges in wireless sensor networks. It is a technique to reduce energy consumption by varying the CPU frequency. Changing the CPU frequency can affect time keeping functionality of sensor platforms. In [9], author stated reduction of energy consumption in wireless sensor networks via dynamic voltage scaling while reducing impact of CPU frequency switching on time synchronization. Dynamic voltage scaling (DVS) and dynamic voltage and frequency scaling (DVFS) techniques have been widely used to reduce energy dissipation in real time and event driven systems.

\subsection{Energy Efficient MAC Protocols}

Wireless sensor network is an attractive choice for various applications as no wired network is involved. Other wireless networks are not as energy constrained as wireless sensor networks. As other networks can be plugged into mains supply or equipped with batteries that are rechargeable and replaceable. One of the prime sources of energy depletion in WSN can be controlled by medium access control(MAC)protocols[10].MAC protocols can be classified as controlled access(CA),random access(RA),slotted $\operatorname{access}(\mathrm{SA})$,hybrid protocols(HP).

\subsection{Energy Aware Routing}

Energy aware data centric routing protocol (EAD) [11] is distributed routing protocol, which forms a virtual backbone comprised of active sensors that are responsible for innetwork data processing and traffic relaying. EAD approach is energy aware and useful extending network lifetime.

\subsection{Duty Cycling}

The most effective energy conserving operation is to put the radio transceiver in low power mode i.e. sleep mode whenever communication between nodes is not necessary. The radio should be switched off as soon as there is no data to transfer or to receive and should be made active as soon as a data packet becomes ready [12]. The switching between active and sleep mode can save energy utilization. In this way, nodes alternate between active and sleep periods.

\subsection{Network Coding}

Network coding is used to enhance a network's throughput, efficiency and scalability [13].This technique improves the capacity of a network with better use of bandwidth. The nodes of the wireless network take several packets and send them together for transmission to attain maximum possible information flow. Intermediate nodes encode data packets received from neighboring nodes. Basically this technique is used to reduce traffic in bottleneck zone or area near the sink. The nodes in bottleneck zone are prone to deplete their energies very quickly called as energy whole problem in WSN. Whenever a node enters into the bottleneck zone, network coding layer maintains received queue and a sensed queue. Whenever it receives a packet a node put the packet in received queue. Encoded (XOR) packet is generated by packets from sensed queue and received queue. If received packet is already processed then it is discarded by the node. If the node is not an encoder node, it acts as relay node and transmit packet to the sink. Duty cycle and network coding technique can be combined to use the network resources effectively.

\section{CONCLUSION}

Energy saving in wireless sensor networks have attracted a lot of attention in the recent years. It is one of the critical aspects for WSNs. The survey is carried out about important approaches to energy conservation in wireless sensor networks. Energy conserving network communication techniques become more important. This paper is survey about what are the techniques that are used in the wireless sensor networks for enhancing the life time of networks. It put forward both advance techniques and some of previous techniques .Important techniques are summarized but still some developments are needed.

\section{REFERENCES}

[1] Römer, Kay; FriedemannMattern "The Design Space of Wireless Sensor Networks" IEEE Wireless Communications, Dec. 2004.

[2] F. Akyildiz, W. Su, Y. Sankarasubramaniam, and E. Cayirci, -Wireless sensor networks: a survey, Computer Networks, vol. 38, no. 4, pp. 393-422, Mar. 2002.

[3] V. Raghunathan, C. Schurgers, S. Park, and M. B. Srivastava, "Energy-Aware wireless Micro sensor Networks", IEEE Signal Processing Magazine, 19 (2002), pp 40-50.

[4] Jamal Al-Karaki, and Ahmed E. Kamal, "Routing Techniques in Wireless Sensor Networks: A Survey“, IEEE Communications Magazine, vol 11, no. 6, Dec. 2004, pp. 6-28.

[5] Kemal Akkaya and Mohamed Younis, "A Survey on Routing Protocols for Wireless Sensor Networks", Ad hoc Networks, vol. 3,no. 3, May 2005, pp. 325-349C

[6] Jun Zheng and Abbas Jamalipour, "Wireless Sensor Networks: A Networking Perspective", a book published by A John \& Sons, Inc, and IEEEE, 2009.

[7] I. Demikol, C Ersoy, F Alagoz, "MAC protocols for wireless Sensor Networks: A survey", Tech. Rep., Bogazici University, Turkey.

[8] P. Minet, "Energy efficient routing", in Ad Hoc and Sensor Wireless Networks: Architectures: Algorithms and Protocols. Bentham Science,2009. 
[9] K. Choi, R. Soma, and M. Pedram. Fine-grained dynamic voltage and frequency scaling for precise energy and performance trade-off based on the ratio of off-chip access to on chip computation times. IEEE Transactions on Computer-Aided Design of Integrated Circuits and Systems, 24(1):18 -28, January 2005.

[10] Qingchun Ren and Qilian Liang "An Energy-Efficient MAC Protocol for Wireless Sensor Networks", Global TelecommunicationsConference, 2005. GLOBECOM'05. IEEE.

[11] A. Boukerche, X. Cheng, and J. Linus, "Energy-aware data-centric routing in micro sensor networks", Proceedings ACM MSWiM, in conjunction with ACM Mobi Com, San Diego, CA, Sept. 2003, pp. 42-49.

[12] Rashmi Ranjan Rout, Soumya K. Ghosh,"Enhancement of Lifetime using Duty Cycle and Network Coding in Wireless Sensor Networks", IEEE Transactions on Wireless Communications, Vol. 12, No. 2, February 2013.

[13] D. Ganesan, A. Cerpa, W. Ye, Y. Yu, J. Zhao, D. Estrin, "Networking Issues in Wireless Sensor Networks", Journal of Parallel and Distributed Computing, Vol. 64 (2004), pp. 799-814.

[14] Karthikkumar, R. Sukumar, "Sensor Lifetime
Enhancement Techniques in Wireless Sensor networks-A Critical Review", IRACST, vol.3. No.2, April 2013.

[15] D. J. Cook and S. K. Das, "Smart environments: technologies, protocols and applications," New York: John Wiley, pp. 13-15, 2004.

[16] Kemal Akkaya and Mohamed Younis, "A Survey on Routing Protocols for Wireless Sensor Networks", Ad hoc Networks, vol. 3, no. 3, May 2005, pp. 325-349.

[17] D. Ganesan, R. Govindan, S. Shenker, D. Estrin, "Highly resilient, energy-efficient multipath routing in wireless sensor networks", ACM SIGMOBILE Mobile Computing and Commun. Rev., Vol. 5, No. 4, pp.11-25, 2001.

[18] K. Sohraby, D. Minoli, T.Znati, "Wireless Sensor Networks: Technology, Protocols, and Applications", 1st ed.; John Wiley and Sons, Inc: Hoboken, NJ, USA, 2007, pp. 38.69

[19] G. Anastasi, M. Conti, M. Di Francesco, \& A. Passarella, -Energy conservation in wireless sensor networks: A surveyll, Ad Hoc Networks, vol. 7, no. 3, pp. 537-568, July 2008

[20] J. Yick, B. Mukherjee, D. Ghosal,"Wireless sensor network survey”, Computer Networks, Vol. 52, No. 12, pp. 2292-2330, 2008. 